\title{
Retrograde radial arteriovenous fistula, post coronary artery intervention
}

\author{
PAWAN GARG ${ }^{1}$, Surendra Patel ${ }^{1}$, Alok Sharma ${ }^{1}$, and Pushpinder Khera ${ }^{1}$ \\ ${ }^{1}$ All India Institute of Medical Sciences Jodphur
}

June 13, 2020

\begin{abstract}
Radial arteriovenous fistula post coronary artery intervention is rare. Here we describe an extremely rare case of radial arteriovenous fistula (AVF) with retrograde filling through the deep palmar arch and presenting as early ischemic changes.
\end{abstract}

\section{Case report:}

A 49-year-old male presented with complaints of mild swelling over the radial aspect of the right distal forearm for the last 2 months with new onset of mild pain in fingertips mainly thumb and index finger for a week (Figure 1A). He had a history of percutaneous transluminal coronary angioplasty (PTCA) 3 months back via the trans-radial route. The swelling started 1 month after the procedure. On examination superficial veins over distal forearm were prominent with thrill was palpable over swelling. Mild prolonged capillary filling time noted mainly of thumb and index fingers with no temperature change, suggestive of early ischemic changes.

Ultrasound followed by computed angiography (CT) angiography was performed for evaluation. CT revealed the complete occlusion of the radial artery from its origin till the distal forearm. The ulnar artery was normal and mild prominent. The radial artery at the wrist and proximal hand was reformed by retrograde flow from the deep palmar arch. This distal radial artery retrogradely supplying the arteriovenous fistula (AVF) at distal forearm (Figure 2A,2B).

Initially, local compression under ultrasound guidance was attempted but not successful. Since the artery was retrogradely filling from palmar arch transarterial embolization and covered stent placement was technically not feasible. Hence surgical ligation of arteriovenous fistula under supraclavicular block was performed (Figure 1B). The proximal and distal right radial artery, cephalic vein, and fistulous connection were identified and the quadruple ligation of the AVF was done. Postoperative recovery was uneventful, and the patient was discharged on the first postoperative day.

\section{Discussion:}

PTCA via trans-radial route is now more common compared to the trans-femoral route in view of less complication and more patient comfort. ${ }^{1}$ Vascular complication includes radial artery occlusion, pseudoaneurysm, and AVF. In the RIVAL study, none out of 3507 patients in trans-radial route developed AVF in the trans-radial route. ${ }^{1,2,3}$ Burzotta et al reported 9 (0.08\%) of the 10676 patients who developed AVF post trans-radial access. ${ }^{1,4}$

AVF can develop due to combined unnoticed puncture of the superficial vein and radial artery during access; however, in most cases the communication seals spontaneously. The factors responsible for radial AVF include less operator experience, multiple puncture attempts, inadequate compression for hemostasis. ${ }^{2}$ The AVF usually remain asymptomatic or present with mild pain and swelling in most of the cases. It can rarely 
lead to ischemic symptoms due to the stealing of blood and high output cardiac failure due to increased venous return.

In our case, possible mechanism is combined puncture of radial artery and vein along with prolonged compression of radial artery proximal to puncture site which stopped antegrade flow but allowed retrograde flow and formation of retrograde AVF.

The techniques that can reduce the vascular complication includes avoiding multiple attempts to access, use sheath size less than the arterial diameter, ultrasound-guided needle placement and optimum compression which stops hemorrhage while allowing normal distal flow. ${ }^{2}$

\section{Conflict of interest}

All the authors declare that there is no conflict of interest. Informed consent was taken from the patient for publication.

\section{Acknowledgment}

None

\section{Author contributions}

PG collected the data and drafted manuscript. SP and AS performed surgical procedure and perioperative management. PG and PK critically revised the manuscript. PG, SP, AS and PK approved the article.

\section{References}

1. Hashimoto S, Shiraishi J, Kimura M, Nishikawa M, Yanagiuchi T, Ito D, Kishita E, Yokoi H, Hyogo M, Shima T, Sawada T. Usefulness of continuous compression using TR Band for radial arteriovenous fistula following trans-radial intervention. Journal of cardiology cases. 2015 Dec 1;12(6):192-4.

2. Dehghani P, Culig J, Patel D, Kraushaar G, Schulte P. Arteriovenous fistula as a complication of transradial coronary angiography: a case report. Journal of medical case reports. 2013 Dec 1;7(1):21.

3. Jolly SS, Yusuf S, Cairns J, Niemela“ K, Xavier D, Widimsky P, Budaj A, Niemela“ M, Valentin V, Lewis BS, Avezum A, Steg PG, Rao SV, Gao P, Afzal R, et al. Radial versus femoral access for coronary angiography and intervention in patients with acute coronary syndromes (RIVAL): a randomized, parallel group, multicenter trial. Lancet 2011;377:1409-20.

4. Burzotta F, Trani C, Mazzari MA, Tommasino A, Niccoli G, Porto I, Leone AM, Tinelli G, Coluccia V, De Vita M, Brancati M, Mongiardo R, Schiavoni G, Crea F. Vascular complications and access crossover in 10,676 transradial percutaneous coronary procedures. Am Heart J 2012;163: 230-8.

\section{Figure legends:}

Figure 1: (A) Clinical images of patient showing prominent superficial veins (white arrow) in the volar aspect of the distal forearm. (B) Intraoperative image showing the arteriovenous fistula site (black arrow).

Figure 2: Computed tomography (CT) angiography of right upper limb 3D images showing prominent ulnar artery (white arrow) forming the palmar arch. Retrograde filling of the distal radial artery in hand (dashed arrow) from deep palmar arch (small white arrows). Further filling of AVF (red arrow) retrogradely at the wrist from the distal radial artery and prominent superficial veins (dashed red arrow). Note is made of non-opacification of the radial artery in the forearm with visualization of paired radial veins (double white arrow). 

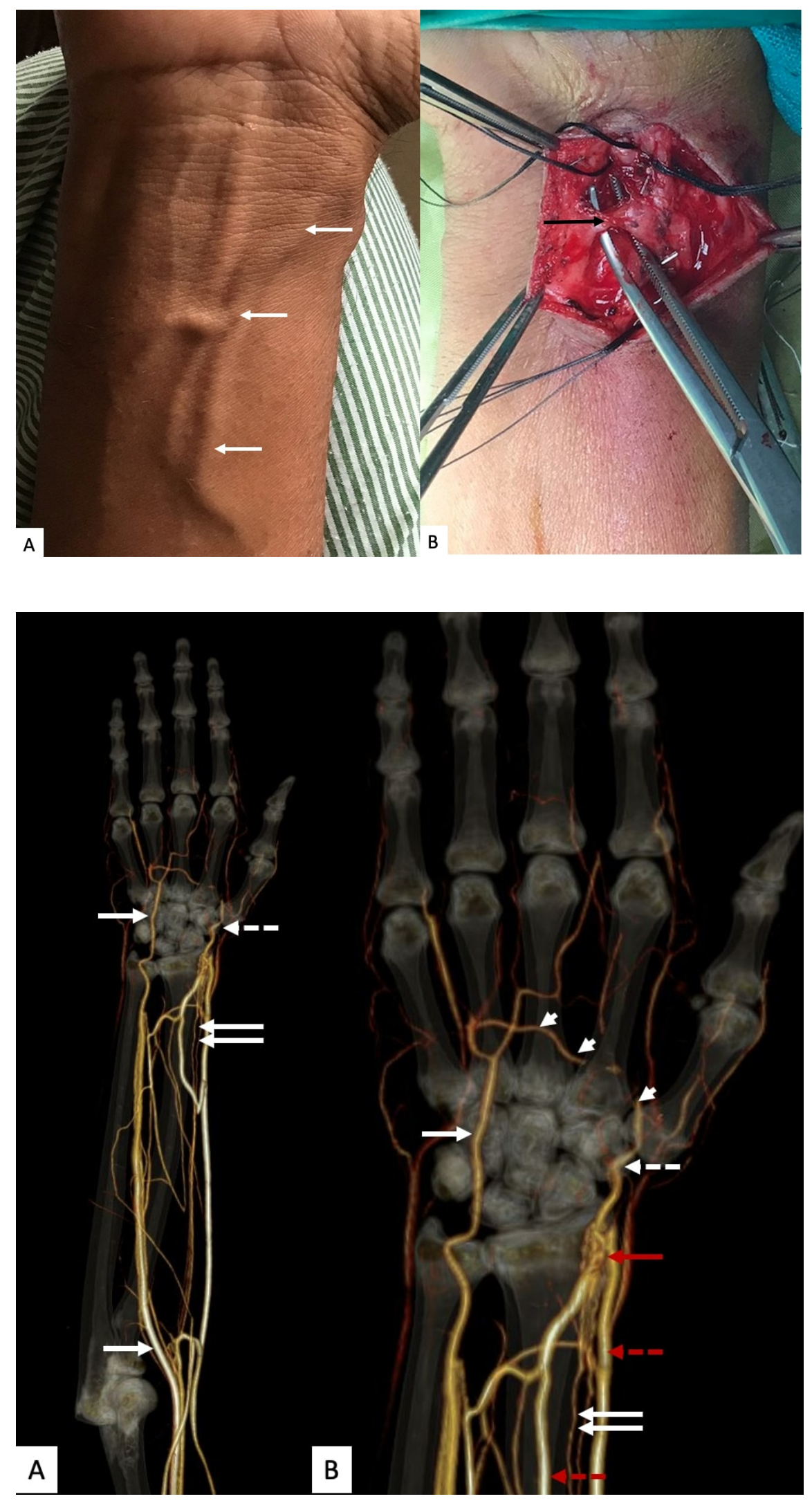